\title{
Dynamics of a particle confined in a two-dimensional dilating and deforming domain
}

\author{
Fabio Anzà, ${ }^{1,2}$ Sara Di Martino, ${ }^{3,4}$ Antonino Messina,,${ }^{1}$ and Benedetto Militello ${ }^{1}$ \\ ${ }^{1}$ Dipartimento di Fisica e Chimica, Università di Palermo, I-90123 Palermo, Italy \\ ${ }^{2}$ Department of Atomic $\& 3$ Laser Physics, Clarendon Laboratory, \\ University of Oxford, Parks Road, Oxford OX1 3PU, UK \\ ${ }^{3}$ Dipartimento di Matematica, Università di Bari, I-70125 Bari, Italy \\ ${ }^{4}$ School of Mathematical Sciences, The University of Nottingham, \\ University Park, Nottingham NG7 2RD, United Kingdom
}

\begin{abstract}
Some recent results concerning a particle confined in a one-dimensional box with moving walls are briefly reviewed. By exploiting the same techniques used for the $1 \mathrm{D}$ problem, we investigate the behavior of a quantum particle confined in a two-dimensional box (a 2D billiard) whose walls are moving, by recasting the relevant mathematical problem with moving boundaries in the form of a problem with fixed boundaries and time-dependent Hamiltonian. Changes of the shape of the box are shown to be important, as it clearly emerges from the comparison between the "pantographic" case (same shape of the box through all the process) and the case with deformation.

PACS numbers: 03.65.-w, 03.65.Db
\end{abstract}

\section{INTRODUCTION}

Over the decades several authors have dealt with the problem of physical systems with moving boundaries, both in quantum field theory, especially in connection with the Casimir effect [1, 2], and in the context of quantum mechanics, for example in connection with the Fermi problem of a quantum bouncer 3]. Over the years, several works appeared studying the problem of particles confined in a box with moving walls [4 [8], sometimes focusing on boundaries having specific shapes 9 -11]. The study of this kind of problems is relevant to several conceptual aspects of quantum mechanics, from the analysis of the semiclassical limit of a quantum (chaotic) system [12 15] to the incoming of geometric phases [16], and it is connected with the derivation of analytical solutions of the dynamics of systems governed by such mathematically complicated potentials as delta functions [17. The interest in such a class of problems is present in different physical scenarios and can lead to intriguing applications. For example, in the field of cavity quantum electrodynamics appeared a study of the implications of small displacements of the mirrors on the dynamics of an atom interacting with the cavity modes [18]. On the other hand, cooling techniques of trapped particles based upon exploitation of expanding boxes have been proposed 19]. Furthermore, the study of two particles in a box with moving boundaries has been recently presented, and the relevant results suggest the idea of an effective interaction between the particles induced by their common interaction with the moving walls [20]. The problem of many particles in a box with moving walls (possibly in higher dimensional situations) has also been treated, especially in connection with shortcuts to adiabaticity and in the context of Bose-Einstein condensates [21, 22].

In this paper, we firstly review some recent results concerning the dynamics of a particle confined in a onedimensional box, pointing out the delicate mathematical aspects of this class of problems and presenting an approach to overcome the relevant difficulties [8]. Secondly, on the basis of this approach, we report on the analytical study of a wide class of problems of a single particle in a two-dimensional box whose walls are moving. In fact, following the way paved in Ref. [9] for a 2D elliptical billiard and in Ref. [8] for a 1D box, we transform the original problem of a system with changing boundaries into the problem of a system with fixed boundaries governed by a time-dependent Hamiltonian. Generally speaking, resolution of dynamical problems in the presence of time-dependent Hamiltonians is a hard task, hence complete or partial analytical solutions are known only in special cases, for example in the adiabatic limit [23] or in the presence of periodical potentials [24 26]. In most cases, perturbation theory [27 30] or numerical methods $31-33]$ are the only effective ways to solve such problems.

In our case, we will exploit standard perturbation approach in order to bring to the light some new effects. We will show that the very novelty in the study of $2 \mathrm{D}$ and $3 \mathrm{D}$ problems with respect to the one-dimensional case is the role of the shape of the moving box, which is obviously absent at all in the one-dimensional case. In fact, for two- and threedimensional boxes, we can consider two classes of problems: on the one hand the ones that we dub as "pantographic", where the dimensions of the box change but its shape remains invariant; on the other hand the cases where the shape (and possibly the dimensions) of the box changes, meaning that we are in the presence of "deformation" of the contour. We start our analysis by constructing the framework of the most general case of a 2D box undergoing dilation and deformation, then we proceed by specializing our analysis to the pantographic case and eventually we present a perturbative treatment of the problem where the shape of the boundary is only slightly modified (small deformations). Though in Refs. [10] and [11] three-dimensional dilating domains have been considered, the role of 
deformation has not been treated in such works. On the contrary, in Ref. [9] it is analyzed an elliptical billiard whose axes have time-dependent lengths, focusing in particular on the case where the lengths of the axes are oscillating at a given frequency, and then numerically evaluating the dynamics of the particle in the moving box. Albeit the authors succeed in going through many details, their analysis is strictly related to a specific geometry. Our work is different since we aim at describing the very more general situation wherein the shape can be modified in any possible way, provided the domain is kept as a star domain. In fact, we will write down the relevant general equations and will study them under the assumption that the deformation can be considered as a perturbation. On this basis, we will evaluate the time evolution through the standard approach in a specific case (a circle which is deformed to an ellipse).

The paper is organized as follows. In the next section we summarize some recent results related to the onedimensional problem, also presenting the general approach of Ref [8] (which is analogous to that of Ref. [9]) that we will use through all the paper. In section III we introduce the problem of a two-dimensional box whose border is changing, defining the two classes of pantographic changes and changes with deformation. In section IV] we study the pantographic case, while in section $\nabla$ we use perturbation theory to study the effects of deformation of the border of the box. Finally in section VI we provide some conclusive remarks and very briefly discuss the extension of the results to the three-dimensional case.

\section{SUMMARY OF PREVIOUS RESULTS: THE ONE-DIMENSIONAL PROBLEM}

\section{A. General framework}

A (free) non-relativistic quantum particle of mass $\mu>0$ confined in a certain domain $\mathcal{D}$ is formally described by the usual (free) particle Hamiltonian in the space region corresponding to the box,

$$
H=-\frac{\hbar^{2}}{2 \mu} \nabla^{2},
$$

imposing suitable boundary conditions, in such a way that $H$ is self-adjoint. One of the possible is that the wave function of the particle is vanishing in the border of the box $(\partial \mathcal{D})$ :

$$
\psi(\mathbf{r})=0, \quad \forall \mathbf{r} \in \partial \mathcal{D} .
$$

Now, if the domain $\mathcal{D}$ is not static, there is a non trivial technical problem in the resolution of the Schrödinger equation related to the fact that we have to solve a differential equation in a Hilbert space which is continuously changing, so that, for example, the time derivative of the wave function is not well defined in the border of the box. Indeed, if $\mathbf{r}_{0}$ is a point of the border at time $t+\mathrm{d} t$ which does not belong to the border at time $t$, then the quantity $\left(\psi\left(\mathbf{r}_{0}, t+\mathrm{d} t\right)-\psi\left(\mathbf{r}_{0}, t\right)\right) / \mathrm{d} t$ is meaningless. According to the approach in Ref [8], this difficulty can be overcome by enlarging the domain of definition of the Hamiltonian in such a way that the such operator acts as the usual (free) particle Hamiltonian in the space region corresponding to the box and is zero elsewhere (i.e., in the complement $\overline{\mathcal{D}}$ ):

$$
H_{\mathcal{D}}=-\frac{\hbar^{2}}{2 \mu} \nabla^{2} \oplus_{\overline{\mathcal{D}}} 0 .
$$

Of course, the condition expressed by Eq. (2) that the wave function of the particle vanishes in the border of the box will be kept. In this way, the wave function turns out to be properly defined for any $\mathbf{r}$, being zero out of the box. Though the most natural choice for the extension of the Hamiltonian would be to put the operator equal to infinity out of the box (which is also a better description from the physical point of view), we stress that here the main reason for extending the Hamiltonian operator is that we want to extend the wave function out of the box. Now, since the wave function is anyway vanishing out of the box because of the boundary conditions, any extension of the Hamiltonian would be fine, in the sense that the specific choice of the form of the operator out of the box is irrelevant to the dynamical description of the system. Therefore, we have decided to exploit the simplest mathematical extension.

In order to better treat the dynamical problem associated to Eq. (3), we can map it into the problem of a particle in a fixed domain, say $\mathcal{D}_{0}$, governed by a suitable effective time-dependent Hamiltonian, $H_{\text {eff }}$ :

$$
\begin{gathered}
H_{\mathcal{D}_{0}}=H_{\text {eff }} \oplus_{\overline{\mathcal{D}}_{0}} 0, \\
\phi(\mathbf{r})=0, \quad \forall \mathbf{r} \in \partial \mathcal{D}_{0} .
\end{gathered}
$$

This passage can be done through the action of a suitable unitary operator. 


\section{B. The one-dimensional box}

In Ref [8] it has been studied the case of a particle in a one-dimensional box when both of its walls are moving, each one with its own velocity; stated another way, the size of the box is changing and at the same time the center of the box is moving. For the sake of simplicity, we will summarize the results when the center of the box is quiet, so that the particle domain is $\mathcal{D}=\left[-R x_{0} / 2, R x_{0} / 2\right]$, with $R$ the dilation dimensionless parameter. This problem is then mapped into the one related to the box delimited by $\mathcal{D}_{0}=\left[-x_{0} / 2, x_{0} / 2\right]$. The relevant transformation is given by the unitary operator $U$ such that

$$
\phi(x)=(U \psi)(x)=R^{1 / 2} \psi(R x) .
$$

Under this transformation, the original Hamiltonian,

$$
H_{\mathcal{D}}=-\frac{\hbar^{2}}{2 \mu} \frac{\partial^{2}}{\partial x^{2}} \oplus_{\overline{\mathcal{D}}} 0
$$

is replaced by the new generator of the time evolution:

$$
H_{\mathcal{D}_{0}}=\left[-\frac{\hbar^{2}}{2 \mu R^{2}} \frac{\partial^{2}}{\partial x^{2}}+\mathrm{i} \hbar \frac{\dot{R}}{R}\left(\frac{1}{2}+x \frac{\partial}{\partial_{x}}\right)\right] \oplus_{\overline{\mathcal{D}}_{0}} 0 .
$$

The price to pay to obtain static boundaries is to make the Hamiltonian explicitly time-dependent. In particular, in this case we have obtained that the kinetic energy, in the new picture, is that of a particle with changing mass (as an effect of the presence of the dilation parameter $R$ in the denominator). Moreover, the particle is subjected to an additional energy term, the one proportional to $\dot{R} / R$, which is nothing but $\mathrm{i} \dot{U} U^{\dagger}$, i.e. the generator of the dilation. It is worth noting that among the two terms, only the first one preserves the physical meaning of energy of the particle, being the result of a unitary transformation of the original energy operator. The second term, instead, is relevant only for determining the dynamics of the particle in the new picture.

Now, since the term $x \partial_{x}$ does not commute with the operator $\partial_{x}^{2}$, the kinetic energy of the particle is not conserved during the process. The relevant energy rate equation, which generalizes and improves the result present in Ref [34], is:

$$
\dot{E}(t)=-\frac{\hbar^{2} \dot{R}}{2 \mu R^{3}}\left[\left|\phi^{\prime}(1 / 2, t)\right|^{2}+\left|\phi^{\prime}(-1 / 2, t)\right|^{2}\right] .
$$

This interesting formula shows that the change of energy is determined by the contact between the particle and the walls. One could think that a natural generalization of this would still be valid in a two- or three-dimensional context. This is what will be shown in the next section, together with some specific properties which have no analogous in the $1 \mathrm{D}$ case.

\section{A QUANTUM PARTICLE IN A TWO-DIMENSIONAL BOX}

In order to start our analysis of a two-dimensional problem, let us consider the case in which the domain of the wave functions is a star domain lying on a plane and delimited by a curve $\gamma$. We recall here that a region $S$ of $\mathbb{R}^{n}$ is said to be a star domain if there exists a point $x_{0} \in S$ such that $\forall x \in S$ the segment $\lambda x_{0}+(1-\lambda) x$, for $\lambda \in[0,1]$, lies in the interior of $S$. Assume that the curve describing the walls of the box can be represented by the following equation:

$$
r=\gamma(\theta), \quad \theta \in[0,2 \pi]
$$

where the origin of the polar coordinates is the center (or one of the possible centers) of the domain. Here the one-to-one correspondence between $r$ and $\theta$ is guaranteed by the property of the domain to have a star-shape. The region delimited by $\gamma$ is expressible as $\mathcal{D}=S_{\gamma}=\{P(r, \theta) \mid r \leq \gamma(\theta), \theta \in[0,2 \pi]\}$. The boundary conditions on the wave function $\psi(r, \theta)$ are expressible as:

$$
\psi(\gamma(\theta), \theta)=0, \quad \forall \theta \in[0,2 \pi] .
$$

The domain $S_{\gamma}$ can be mapped to another domain $\left(\mathcal{D}_{0}=S_{\eta}=\{P(r, \theta) \mid r \leq \eta(\theta), \theta \in[0,2 \pi])\right.$ by the transformation $U$ acting as follows:

$$
\phi(s, \theta)=(U \psi)(s, \theta)=R(\theta) \psi(s R(\theta), \theta), \quad R(\theta)=\gamma(\theta) / \eta(\theta) .
$$


Such transformation is unitary. Indeed,

$$
\begin{aligned}
\int_{S_{\eta}} \phi_{1}^{*}(s, \theta) \phi_{2}(s, \theta) s \mathrm{~d} s \mathrm{~d} \theta & =\int_{S_{\eta}} R(\theta)^{2} \psi_{1}^{*}(s R(\theta), \theta) \psi_{2}(s R(\theta), \theta) s \mathrm{~d} s \mathrm{~d} \theta \\
& =\int_{S_{\gamma}} \psi_{1}^{*}(r, \theta) \psi_{2}(r, \theta) r \mathrm{~d} r \mathrm{~d} \theta
\end{aligned}
$$

If the boundary is moving, we have that the curve $\gamma$ changes and then the function $R$ depends on $t, R(\theta, t)$.

Now, for the 2D problem of a confined particle, we have:

$$
\begin{aligned}
& H=\frac{p^{2}}{2 \mu}=-\frac{\hbar^{2}}{2 \mu} \nabla^{2} \\
& \nabla^{2}=\left(\frac{1}{r} \frac{\partial}{\partial r}+\frac{\partial^{2}}{\partial r^{2}}+\frac{1}{r^{2}} \frac{\partial^{2}}{\partial \theta^{2}}\right) \\
& \left(U^{\dagger} \phi\right)(r, \theta)=\frac{1}{R(\theta, t)} \phi(r / R(\theta, t), \theta), \\
& \left(\frac{\mathrm{d} U}{\mathrm{~d} t} \psi\right)(r, \theta)=\frac{\mathrm{d}}{\mathrm{d} t}(U \psi)(r, \theta)= \\
& =\frac{\mathrm{d}}{\mathrm{d} t}[R(\theta, t) \psi(r R(\theta, t), \theta)]=\dot{R}(\theta, t) \psi(r R(\theta, t), \theta)+R(\theta, t) r \dot{R}(\theta, t) \psi_{1}(r R(\theta, t), \theta) \\
& =\dot{R}(\theta, t)\left[\psi(r R(\theta, t), \theta)+r R(\theta, t) \psi_{1}(r R(\theta, t), \theta)\right] \text {, }
\end{aligned}
$$

where $\psi_{k}$ is the derivative with respect to the $k$-th argument. Then,

$$
\begin{aligned}
\mathrm{i} \hbar\left(\frac{\mathrm{d} U}{\mathrm{~d} t}\left(U^{\dagger} \phi\right)\right)(r, \theta) & =\mathrm{i} \hbar \frac{\mathrm{d} U}{\mathrm{~d} t}\left[\frac{1}{R(\theta, t)} \phi(r / R(\theta, t), \theta)\right] \\
& =\mathrm{i} \hbar \frac{\dot{R}(\theta, t)}{R(\theta, t)}\left[\phi(r, \theta)+r \phi_{1}(r, \theta)\right] \\
& =\mathrm{i} \hbar \frac{\dot{R}(\theta, t)}{R(\theta, t)}\left(1+r \frac{\partial}{\partial r}\right) \phi(r, \theta) \\
U H U^{\dagger} \phi(r, \theta)= & -\frac{\hbar^{2}}{2 \mu} U\left(\frac{1}{r} \frac{\partial}{\partial r}+\frac{\partial^{2}}{\partial r^{2}}+\frac{1}{r^{2}} \frac{\partial^{2}}{\partial \theta^{2}}\right)^{\frac{1}{R}} \phi(r / R, \theta) \\
= & -\frac{\hbar^{2}}{2 \mu}\left\{\frac{1}{r R^{2}} \frac{\partial}{\partial r} \phi(r, \theta)+\frac{1}{R^{2}} \frac{\partial^{2}}{\partial r^{2}} \phi(r, \theta)+\frac{1}{r^{2} R^{2}} \frac{\partial^{2}}{\partial \theta^{2}} \phi(r, \theta)\right. \\
+ & \left.\frac{1}{R}\left(\frac{1}{R}\right)_{\theta \theta} \frac{1}{r^{2}} \phi(r, \theta)+\left[\left(\frac{1}{R}\right)_{\theta}\right)^{2}+\left(\frac{1}{R}\left(\frac{1}{R}\right)_{\theta}\right)_{\theta}\right] \frac{1}{r} \frac{\partial}{\partial r} \phi(r, \theta) \\
+ & \left.\frac{2}{R}\left(\frac{1}{R}\right)_{\theta} \frac{1}{r^{2}} \frac{\partial}{\partial \theta} \phi(r, \theta)+\left[\left(\frac{1}{R}\right)_{\theta}\right]^{2} \frac{\partial^{2}}{\partial r^{2}} \phi(r, \theta)+\frac{2}{R}\left(\frac{1}{R}\right)_{\theta} \frac{1}{r} \frac{\partial}{\partial r} \frac{\partial}{\partial \theta} \phi(r, \theta)\right\}
\end{aligned}
$$

where the subindex $\theta$ means derivative with respect to $\theta$. It is worth mentioning two technical aspects relevant to the previous equations. First, note that the wave functions $\psi$ and $\phi$ are assumed as time-independent. Indeed, they are not meant as solutions of the Schrödinger equation, but as generic elements of the Hilbert spaces to which they belong. We just use them to make explicit the action of the operators considered above. Second, when we perform $\theta$-derivatives, we have to take care of the twofold dependence on $\theta$ of the wave function, due to both the 'natural' $\theta$-dependence and the possible additional $\theta$-dependence due to the action of $U$ or $U^{\dagger}$. For example, $\partial_{\theta} U^{\dagger} \phi(r, \theta)=\partial_{\theta}\left[R^{-1} \phi\left(R^{-1} r, \theta\right)\right]$, where $R$ in general is a function of $\theta$. 
The effective Hamiltonian associated to the problem in the fixed domain $-H_{\mathrm{eff}}=U H U^{\dagger}+\mathrm{i} \hbar \dot{U} U^{\dagger}-$ is rather involved, due to the deformation of the boundary. In fact, such Hamiltonian can be considered as the sum of three terms:

$$
\begin{aligned}
H_{\mathrm{eff}} & =H_{1}+H_{2}+H_{3} \\
H_{1} & =-\frac{\hbar^{2}}{2 \mu R^{2}} \nabla^{2} \\
H_{2} & =\mathrm{i} \hbar \frac{\dot{R}}{R}\left(1+r \frac{\partial}{\partial r}\right) \\
H_{3} & =-\frac{\hbar^{2}}{2 \mu}\left\{\frac{1}{R}\left(\frac{1}{R}\right)_{\theta \theta} \frac{1}{r^{2}}+\left[\left(\left(\frac{1}{R}\right)_{\theta}\right)^{2}+\left(\frac{1}{R}\left(\frac{1}{R}\right)_{\theta}\right)_{\theta}\right] \frac{1}{r} \frac{\partial}{\partial r}\right. \\
& \left.+\frac{2}{R}\left(\frac{1}{R}\right)_{\theta} \frac{1}{r^{2}} \frac{\partial}{\partial \theta}+\left[\left(\frac{1}{R}\right)_{\theta}\right]^{2} \frac{\partial^{2}}{\partial r^{2}}+\frac{2}{R}\left(\frac{1}{R}\right)_{\theta} \frac{1}{r} \frac{\partial}{\partial r} \frac{\partial}{\partial \theta}\right\} .
\end{aligned}
$$

It is worth noting that when the relation between the moving domain $\mathcal{D}$ and the static domain $\mathcal{D}_{0}$ is pantographic, which means that they have the same shape, the function $R(\theta, t)$ does not really depend on $\theta$ and all the terms in $H_{3}$ vanish. On the contrary, if there is a deformation of the domain, $R_{\theta}$ is non zero and $H_{3}$ is non vanishing.

We interpret and address the terms in $H_{3}$ as deformation terms. The remaining contributions, in the case $R_{\theta}=0$, give what we call the pantographic Hamiltonian (well defined in the beginning of next section). In this situation, the term $H_{1}$ can be interpreted as the Hamiltonian of a particle with a varying mass, while $H_{2}$ is the dilation term. It is anyway important to note that in the presence of deformation also $H_{1}+H_{2}$ depends on $\theta$, hence giving rise to additional deformation terms. In fact, all the terms in $H_{3}$ are associated to deformation, but there are deformation terms which do not belong to $H_{3}$. This will be clearer in section $\nabla$ where the problem of deformation will be treated extensively. Instead, in the next section we will consider only pantographic changes of the domain, hence assuming $R_{\theta}=0$ and $H_{3}=0$.

\section{THE PANTOGRAPHIC CASE}

We call pantographic Hamiltonian the operator

$$
H_{\mathrm{p}}^{\lambda}=-\frac{\hbar^{2}}{2 \mu \lambda^{2}} \nabla^{2}+\mathrm{i} \hbar \frac{\dot{\lambda}}{\lambda}\left(1+r \frac{\partial}{\partial r}\right), \quad \partial_{\theta} \lambda=0,
$$

i.e. the remaining part of the Hamiltonian in the absence of deformation, provided $R(t)=\lambda(t)$.

In such a case, the pantographic Hamiltonian governs the dynamics of the particle in the picture associated to the fixed domain $\mathcal{D}_{0}$. In this simplified situation it is possible to deduce a rate equation for the energy of the particle and to derive the exact dynamics in the special case of constant speed of the walls.

\section{A. The Energy rate equation}

Let us now consider the energy rate equation in the two-dimensional pantographic case. In the original picture (with moving walls), the average energy of the system is given by $E(t)=\langle\psi|H| \psi\rangle$, so that in the picture with static walls such average energy is given by the mean value, over the state $|\phi\rangle$, of the operator $U H U^{\dagger}=H_{1}+H_{3}$ in Eq. (19), from which we have to cancel the deformation terms, hence obtaining $U H U^{\dagger}=H_{1}$.

The energy rate equation is then given by:

$$
\dot{E}(t)=\frac{\mathrm{d}}{\mathrm{d} t}\left\langle\phi\left|H_{1}\right| \phi\right\rangle=\frac{i}{\hbar}\left(\left\langle\phi \mid H_{2} H_{1} \phi\right\rangle-\left\langle H_{2} H_{1} \phi \mid \phi\right\rangle\right)+\left\langle\phi\left|\dot{H}_{1}\right| \phi\right\rangle,
$$

with

$$
\dot{H}_{1}=\frac{\hbar^{2} \dot{R}}{\mu R^{3}} \nabla^{2}
$$


It is just the case to mention that the operator

$$
H_{2}=\mathrm{i} \hbar \frac{\dot{R}}{R}\left(1+r \frac{\partial}{\partial r}\right)=\mathrm{i} \hbar \frac{\dot{R}}{R}\left(\frac{1}{2}+r \circ \frac{\partial}{\partial r}\right)
$$

where $A \circ B=(A B+B A) / 2$ is the Jordan product of the operators $A$ and $B$, has domain

$$
D\left(H_{2}\right)=\left\{f \in \mathcal{H}^{1}\left(\mathbb{R}^{2}\right), r \frac{\partial}{\partial r} f(r, \theta) \in L^{2}\left(\mathbb{R}^{2}\right)\right\},
$$

while the product $H_{2} H_{1}$ has domain:

$$
D\left(H_{2} H_{1}\right)=\left\{f \in \mathcal{H}^{3}\left(\mathcal{D}_{0}\right), f(\mathbf{r})=0 \forall \mathbf{r} \in \partial \mathcal{D}_{0}\right\} .
$$

Here we denoted with $\mathcal{H}^{1}$ the first Sobolev space, i.e. $\mathcal{H}^{1}\left(\mathbb{R}^{2}\right)=\left\{f \in L^{2}\left(\mathbb{R}^{2}\right) \mid f^{\prime} \in L^{2}\left(\mathbb{R}^{2}\right)\right\}$, and with $\mathcal{H}^{3}$ the third Sobolev space, i.e. the space of square integrable functions with square integrable third derivative.

After introducing $A \equiv r \partial_{r}$, we can write the rate equation for the energy in the following way:

$$
\dot{E}(t)=\frac{\hbar^{2} \dot{R}}{2 \mu R^{3}} \int_{\mathcal{D}_{0}} r\left[\phi^{*}(1+A) \nabla^{2} \phi+\phi(1+A) \nabla^{2} \phi^{*}+2 \phi^{*} \nabla^{2} \phi\right] \mathrm{d} r \mathrm{~d} \theta .
$$

Since the following relations hold, $[A, \vec{\nabla}]=-\vec{\nabla},\left[A, \nabla^{2}\right]=-2 \nabla^{2}, \phi^{*} A \nabla^{2} \phi=\phi^{*} \nabla^{2} A \phi-2 \phi^{*} \nabla^{2} \phi, \phi A \nabla^{2} \phi^{*}=$ $\phi \nabla^{2} A \phi^{*}-2 \phi \nabla^{2} \phi^{*}$, the terms inside the square brackets of Eq. (30) can be rearranged as $\vec{\nabla} \cdot\left(\phi^{*} \vec{\nabla} \phi-\phi \vec{\nabla} \phi^{*}\right)+$ $\phi^{*} \nabla^{2} A \phi+\phi \nabla^{2} A \phi^{*}$.

Now, on the basis of the Gauss-Green theorem and the fact that the wave function vanishes on the boundary, we can assert that the divergence does not contribute to the integral in Eq. (30). Therefore, we reach the equation

$$
\dot{E}(t)=\frac{\hbar^{2} \dot{R}}{2 \mu R^{3}} \int_{\mathcal{D}_{0}} r\left(\phi^{*} \nabla^{2} A \phi+\phi \nabla^{2} A \phi^{*}\right) \mathrm{d} r \mathrm{~d} \theta .
$$

By exploiting the Leibniz rule we obtain $\phi^{*} \nabla^{2} A \phi=\vec{\nabla} \cdot\left(\phi^{*} \vec{\nabla} A \phi\right)-\left(\vec{\nabla} \phi^{*}\right) \cdot(\vec{\nabla}(A \phi))$ and $\phi \nabla^{2} A \phi^{*}=\vec{\nabla} \cdot\left(\phi \vec{\nabla} A \phi^{*}\right)-$ $\underset{\text { as: }}{(\vec{\nabla} \phi) \cdot\left(\vec{\nabla}\left(A \phi^{*}\right)\right) \text {, which, using again the Gauss-Green theorem, allow to further rearrange the energy rate equation }}$

$$
\dot{E}(t)=\frac{\hbar^{2} \dot{R}}{2 \mu R^{3}} \int_{\mathcal{D}_{0}} r\left(-\left(\vec{\nabla} \phi^{*}\right) \cdot(\vec{\nabla}(A \phi))-(\vec{\nabla} \phi) \cdot\left(\vec{\nabla}\left(A \phi^{*}\right)\right)\right) \mathrm{d} r \mathrm{~d} \theta .
$$

Since $\vec{\nabla}(A \phi)=A \vec{\nabla} \phi+[\vec{\nabla}, A] \phi=A \vec{\nabla} \phi+\vec{\nabla} \phi$, we get

$$
\dot{E}(t)=\frac{\hbar^{2} \dot{R}}{2 \mu R^{3}} \int_{\mathcal{D}_{0}} r\left[-A\left(\|\vec{\nabla} \phi\|^{2}\right)-2\|\vec{\nabla} \phi\|^{2}\right] \mathrm{d} r \mathrm{~d} \theta .
$$

Moreover, integration by part of the first term in the square brackets gives

$$
\int_{\mathcal{D}_{0}} r A\|\vec{\nabla} \phi\|^{2} \mathrm{~d} r \mathrm{~d} \theta=\int_{\theta} \int_{r} r^{2} \partial_{r}\|\vec{\nabla} \phi\|^{2} \mathrm{~d} r \mathrm{~d} \theta=\int_{0}^{2 \pi} d \theta\left[r^{2}\|\vec{\nabla} \phi\|^{2}\right]_{r=0}^{r=\eta(\theta)}-2 \int_{\mathcal{D}_{0}} r\|\vec{\nabla} \phi\|^{2} \mathrm{~d} r \mathrm{~d} \theta
$$

and we eventually get

$$
\dot{E}(t)=-\left.\frac{\hbar^{2} \dot{R}}{2 \mu R^{3}} \int_{0}^{2 \pi} \mathrm{d} \theta\left(r^{2}\|\vec{\nabla} \phi\|^{2}\right)\right|_{r=\eta(\theta)} .
$$

It is easy to see that this is the natural $2 D$ extension of the contact term that appears in the one-dimensional case, according to the analysis developed in Ref [8] . 


\section{B. The case of a uniformly moving domain}

Let us consider now the special case of a pantographic change with walls moving at constant velocity, which means $R=1+\kappa t$. In this situation we are able to completely solve the dynamics of the system by generalizing the results in Ref [4].

Assume that a solution of the time-dependent Schrödinger equation is given by a state with the following form:

$$
\phi_{n}(r, \theta)=e^{\mathrm{i} P_{n}(r, t)} \chi_{n}(r, \theta),
$$

where $P=\alpha_{n}(t) r^{2}+\beta_{n}(t)$ is a polynomial of $r$ with time-dependent coefficients and $\chi_{n}$ is a solution of the eigenvalue problem $-\hbar^{2} /(2 \mu) \nabla^{2} \chi_{n}=E_{n} \chi_{n}$, with $\phi_{n}, \chi_{n} \in L^{2}\left[\mathcal{D}_{0}\right], \phi_{n}(\eta(\theta), \theta)=\chi_{n}(\eta(\theta), \theta)=0$.

We will show that for a suitable choice of $\alpha_{n}$ and $\beta_{n}$ one finds a solution of the Schrödinger equation. In fact, since

$$
\begin{aligned}
\nabla P_{n} & =\frac{\partial P_{n}}{\partial r} \hat{r}=2 \alpha_{n} r \hat{r}, \\
r \frac{\partial P_{n}}{\partial r} & =2 \alpha_{n} r^{2}, \\
\nabla^{2} P_{n} & =\frac{1}{r} \frac{\partial P_{n}}{\partial r}+\frac{\partial^{2} P_{n}}{\partial r^{2}}=4 \alpha_{n},
\end{aligned}
$$

the Schrödinger equation,

$$
\mathrm{i} \hbar \partial_{t} \phi+H_{\mathrm{eff}} \phi=0
$$

expressible as,

$$
\left\{-\hbar \dot{P}_{n} \chi+\frac{\hbar^{2}}{2 \mu R^{2}}\left[\left(\mathrm{i}\left(\nabla^{2} P_{n}\right)-\left(\nabla P_{n}\right)^{2}\right) \chi_{n}+2 \mathrm{i} \frac{\partial P_{n}}{\partial r} \frac{\partial \chi_{n}}{\partial r}+\nabla^{2} \chi_{n}\right]-\mathrm{i} \hbar \frac{\dot{R}}{R} \chi_{n}+\hbar \frac{\dot{R}}{R} r \frac{\partial P_{n}}{\partial r} \chi_{n}-\mathrm{i} \hbar \frac{\dot{R}}{R} r \frac{\partial \chi_{n}}{\partial r}\right\} e^{\mathrm{i} P_{n}}=0
$$

leads to,

$$
\left[-\hbar \dot{\alpha_{n}} r^{2}-\hbar \dot{\beta_{n}}+\frac{\hbar^{2}}{2 \mu R^{2}}\left(4 \mathrm{i} \alpha_{n}-4 \alpha_{n}^{2} r^{2}\right)-\frac{E_{n}}{R^{2}}-\mathrm{i} \hbar \frac{\dot{R}}{R}+2 \hbar \frac{\dot{R}}{R} \alpha_{n} r^{2}\right] \chi_{n} e^{\mathrm{i} P_{n}}+\left[\frac{\mathrm{i} \hbar^{2}}{\mu R^{2}} \frac{\partial P_{n}}{\partial r}-\mathrm{i} \hbar \frac{\dot{R}}{R} r\right] \frac{\partial \chi_{n}}{\partial r} e^{\mathrm{i} P_{n}}=0 .
$$

By imposing the coefficient of $\partial \chi_{n} / \partial r$ equal to zero, one gets

$$
\alpha_{n}=\frac{\mu}{2 \hbar} R \dot{R} \equiv \alpha
$$

By substituting this result in the coefficient of $\chi_{n}$, and imposing it to be zero, one gets:

$$
\left(-\hbar \dot{\beta_{n}}-\frac{E_{n}}{R^{2}}\right)-\frac{\mu}{2} R \ddot{R} r^{2}=0
$$

which, in the case $\ddot{R}=0$, gives rise to the solution:

$$
\beta_{n}=\beta_{n}(0)-\int_{0}^{t} \frac{E_{n}}{\hbar R^{2}(s)} \mathrm{d} s .
$$

The solution of the Schrödinger equation in the original domain $\mathcal{D}$ is given by

$$
\psi_{n}(r, \theta, t)=R^{-1} \exp \left(\mathrm{i} \beta_{n}(t)+\mathrm{i} \alpha(t)(r / R)^{2}\right) \chi_{n}(r / R, \theta) .
$$

Note that $\beta_{n}$ depends on the eigenvalue $E_{n}$ associated to the state $\chi_{n}$, while $\alpha_{n}$ is the same for all $\chi_{n}$ 's.

Therefore one has

$$
\begin{aligned}
\int_{\mathcal{D}} \psi_{k}^{*}(r, \theta, t) \psi_{l}(r, \theta, t) r \mathrm{~d} r \mathrm{~d} \theta & =\int_{\mathcal{D}} R^{-2} e^{\mathrm{i}\left(\beta_{l}(t)-\beta_{k}(t)\right)} \chi_{k}^{*}(r / R, \theta) \chi_{l}(r / R, \theta) r \mathrm{~d} r \mathrm{~d} \theta \\
& =e^{\mathrm{i}\left(\beta_{l}(t)-\beta_{k}(t)\right)} \int_{\mathcal{D}_{0}} \chi_{k}^{*}(s, \theta) \chi_{l}(s, \theta) s \mathrm{~d} s \mathrm{~d} \theta=\delta_{k l}
\end{aligned}
$$


Then, the $\psi_{k}$ 's are an orthonormal set. Moreover, they constitute a basis. To prove this statement, consider the following. It is immediate to prove that since $\chi_{k}(r, \theta)$ 's are a basis for $L^{2}\left[\mathcal{D}_{0}\right]$ then $R^{-1} \chi_{k}(r / R, \theta)$ 's are a basis for $L^{2}[\mathcal{D}]$ - in both cases with the appropriate boundary conditions. Now, given any function in $\xi(r, \theta) \in L^{2}[\mathcal{D}]$ satisfying the boundary condition $\xi(R \eta(\theta), \theta)=0$, one can consider the function $e^{-\mathrm{i} \alpha(t)(r / R)^{2}} \xi(r, \theta)$ and expand it, at time $t$, in terms of the $R^{-1} \chi_{k}(r / R, \theta)$ 's as follows:

$$
e^{-\mathrm{i} \alpha(t)(r / R)^{2}} \xi(r, \theta)=\sum_{k} c_{k} \chi_{k}(r / R, \theta)=\sum_{k} \tilde{c}_{k} e^{\mathrm{i} \beta_{k}(t)} R^{-1} \chi_{k}(r / R, \theta),
$$

and then,

$$
\xi(r, \theta)=\sum_{k} \tilde{c}_{k} e^{\mathrm{i} \alpha(t)(r / R)^{2}} e^{\mathrm{i} \beta_{k}(t)} R^{-1} \chi_{k}(r / R, \theta)=\sum_{k} \tilde{c}_{k} \psi_{k}(r, \theta, t),
$$

which completes the proof that the $\psi_{k}$ 's are a basis.

\section{PERTURBATIVE TREATMENT OF DEFORMATIONS}

In this section we try to analyze the case in which deformations are present. We will concentrate to small deformations, since they can be treated with a standard time-dependent perturbation approach.

\section{A. General Framework}

Assume $R(\theta, t)=\lambda(t)[1+\epsilon f(\theta, t)]$, where $\epsilon$ plays the role of a deformation parameter $(\epsilon=0$ means that no deformation is present). In view of a perturbative treatment, we first assume that $f(\theta, t)$ is a smooth function and that $\epsilon \ll 1$ (in the end of this section we comment on such assumptions). Then we consider series expansions of all relevant functions truncated to the first order in $\epsilon: R^{-2} \approx \lambda^{-2}(1-2 \epsilon f), \dot{R} / R \approx \lambda^{-1} \dot{\lambda}+\epsilon \dot{f}, R^{-1} \approx \lambda^{-1}(1-\epsilon f)$, $\partial_{\theta} R^{-1} \approx-\epsilon \lambda^{-1} \partial_{\theta} f, \partial_{\theta}^{2} R^{-1} \approx-\epsilon \lambda^{-1} \partial_{\theta}^{2} f$. Consequently, we evaluate the first order terms of the total Hamiltonian $H_{\text {eff }}$ :

$$
\begin{gathered}
H_{1}=-\frac{\hbar^{2}}{2 \mu \lambda^{2}} \nabla^{2}+\frac{\hbar^{2} \epsilon f}{\mu \lambda^{2}} \nabla^{2}+O\left(\epsilon^{2}\right), \\
H_{2}=\mathrm{i} \hbar \frac{\dot{\lambda}}{\lambda}\left(1+r \frac{\partial}{\partial r}\right)+\mathrm{i} \hbar \epsilon \dot{f}\left(1+r \frac{\partial}{\partial r}\right)+O\left(\epsilon^{2}\right), \\
H_{3}=-\epsilon \lambda^{-2}\left(-\frac{\hbar^{2}}{2 \mu}\right)\left[\left(\partial_{\theta}^{2} f\right)\left(\frac{1}{r^{2}}+\frac{1}{r} \frac{\partial}{\partial r}\right)+2\left(\partial_{\theta} f\right)\left(\frac{1}{r^{2}} \frac{\partial}{\partial \theta}+\frac{1}{r} \frac{\partial}{\partial r} \frac{\partial}{\partial \theta}\right)\right]+O\left(\epsilon^{2}\right) \\
=\epsilon \frac{\hbar^{2}}{2 \mu} \lambda^{-2}\left[\partial_{\theta}^{2} f+2\left(\partial_{\theta} f\right) \frac{\partial}{\partial \theta}\right]\left(\frac{1}{r^{2}}+\frac{1}{r} \frac{\partial}{\partial r}\right)+O\left(\epsilon^{2}\right),
\end{gathered}
$$

so that the zeroth order Hamiltonian is $H_{\mathrm{eff}}^{(0)}=H_{\mathrm{p}}^{\lambda}$, as defined in Eq. (24), while the first order terms are gathered as

$$
H_{\mathrm{eff}}^{(1)}=\epsilon\left\{\frac{\hbar^{2}}{\mu} \lambda^{-2} f \nabla^{2}+\mathrm{i} \hbar \dot{f}\left(1+r \frac{\partial}{\partial r}\right)+\frac{\hbar^{2}}{2 \mu} \lambda^{-2}\left[\partial_{\theta}^{2} f+2\left(\partial_{\theta} f\right) \frac{\partial}{\partial \theta}\right]\left(\frac{1}{r^{2}}+\frac{1}{r} \frac{\partial}{\partial r}\right)\right\}
$$

Since $\epsilon \ll 1$, we can start by considering the pantographic dynamics with dilation function $\lambda(t)$ as the unperturbed dynamics, and then slightly correct the solutions of the pantographic problem to obtain a first order approximation of the solutions of the problem with small deformation. In fact, by applying the standard time-dependent perturbation theory approach [35], after introducing the unitary operator $U_{\mathrm{p}}$ such that $\mathrm{i} \partial_{t} U_{\mathrm{p}}=H_{\mathrm{p}}^{\lambda} U_{\mathrm{p}}$, we get:

$$
|\psi(t)\rangle=U^{\dagger} U_{\mathrm{p}}(t)\left[1-\mathrm{i} \hbar \int_{0}^{t} U_{\mathrm{p}}^{\dagger}(s) H_{\mathrm{eff}}^{(1)}(s) U_{\mathrm{p}}(s) \mathrm{d} s\right]|\psi(0)\rangle+O\left(\epsilon^{2}\right),
$$


where $U$ is the previously introduced unitary transformation that maps the moving domain into the static one and where we have exploited the fact that $|\phi(0)\rangle=|\psi(0)\rangle$. Moreover, by introducing unity operators we get the following:

$$
\begin{aligned}
|\psi(t)\rangle & =\sum_{\sigma} a_{\sigma}(0)\left|\psi_{\sigma}(t)\right\rangle-\mathrm{i} \hbar \sum_{\sigma}\left|\psi_{\sigma}(t)\right\rangle \int_{0}^{t}\left\langle\psi_{\sigma}(0)\left|U_{\mathrm{p}}^{\dagger}(s) U U^{\dagger} H_{\mathrm{eff}}^{(1)}(s) U U^{\dagger} U_{\mathrm{p}}(s) \sum_{\sigma^{\prime}} a_{\sigma^{\prime}}(0)\right| \psi_{\sigma^{\prime}}(0)\right\rangle \mathrm{d} s+O\left(\epsilon^{2}\right) \\
& =\sum_{\sigma} a_{\sigma}(0)\left|\psi_{\sigma}(t)\right\rangle-\mathrm{i} \hbar \sum_{\sigma}\left\{\sum_{\sigma^{\prime}} a_{\sigma^{\prime}}(0) \int_{0}^{t}\left\langle\psi_{\sigma}(s)\left|U^{\dagger} H_{\mathrm{eff}}^{(1)}(s) U\right| \psi_{\sigma^{\prime}}(s)\right\rangle \mathrm{d} s\right\}\left|\psi_{\sigma}(t)\right\rangle+O\left(\epsilon^{2}\right) \\
& =\sum_{\sigma} a_{\sigma}(0)\left|\psi_{\sigma}(t)\right\rangle-\mathrm{i} \hbar \sum_{\sigma}\left\{\sum_{\sigma^{\prime}} a_{\sigma^{\prime}}(0) \int_{0}^{t}\left\langle\phi_{\sigma}(s)\left|H_{\mathrm{eff}}^{(1)}(s)\right| \phi_{\sigma^{\prime}}(s)\right\rangle \mathrm{d} s\right\}\left|\psi_{\sigma}(t)\right\rangle+O\left(\epsilon^{2}\right),
\end{aligned}
$$

where $\sigma$ and $\sigma^{\prime}$ denote suitable sets of quantum numbers, and $a_{\sigma}(0)=\left\langle\psi_{\sigma}(0) \mid \psi(0)\right\rangle$.

It is necessary to stress here the importance of evaluating the action of $U$ exactly, in order to prevent violation of the boundary conditions.

Moreover, at this stage, it is worthy to spend some words about the validity of our perturbation treatment itself, since one could wonder whether all the neglected terms of the Hamiltonian and the discarded terms in the perturbative expansion are really negligible. A positive answer is based on the treatment of boundary perturbation shortly reported in [36], where it is introduced the problem of a "free" particle in a box whose shape is slightly modified. According to Kato, provided the deformation is "small" and "smooth", Taylor expansion of the eigenvalues and eigenvectors is justified and perturbation treatment is legitimated. In our case, we have two additional ingredients: (a) a dilation is also considered, not simply a deformation, and (b) the perturbation is time dependent; but none of such two elements introduce significant differences. In fact, the presence of the dilation implies that the condition of "smallness" is strictly fulfilled provided $\epsilon \lambda(t) \sup _{\theta} f(\theta, t) \ll 1 \forall t$, while the "smoothness" is guaranteed by the smoothness of the deformation function $f(\theta, t)$ with respect to $\theta$. Finally, the time dependence implies the presence of a transport term $\left(\mathrm{i} \hbar \dot{U} U^{\dagger}\right)$, whose structure, in the specific case, gives rise to the dilation term $\left(\propto \alpha(\theta)\left(1+r \partial_{r}\right)\right)$, and again, following the line of [36] it turns out that, under the assumption of smooth and small deformation, one easily shows that this additional term does not compromise the possibility of Taylor-expanding the eigensolutions and to resort to perturbation treatment.

\section{B. The case of an elliptical box}

As an example, let us consider a circular box which expands and squeezes becoming an ellipse.

The moving and static domain are:

$$
\begin{gathered}
\mathcal{D}=\left\{(r, \theta): r \leq r_{0} \lambda(t) /(1-\epsilon g(t) \cos \theta), \theta \in[0,2 \pi]\right\}, \quad \epsilon \ll 1, \\
\mathcal{D}_{0}=\left\{(r, \theta): r \leq r_{0}, \theta \in[0,2 \pi]\right\},
\end{gathered}
$$

where $\lambda(t)$ and $g(t)$ are suitable functions such that $\lambda(0)=1$ and $g(0)=0$ (at $t=0$ the domain $\mathcal{D}$ coincides with $\mathcal{D}_{0}$ ), and $g(t)$ tends toward unity (after the value of $g(t)$ stabilizes the shape of $\mathcal{D}$ does not significantly change). Here $\epsilon$ is the asymptotic value of the eccentricity of the time-changing ellipse, and since it is much smaller than unity then we get:

$$
\begin{gathered}
R(\theta, t)=\frac{\lambda(t)}{1-\epsilon f(\theta, t)} \approx \lambda(t)[1+\epsilon f(\theta, t)], \quad f(\theta, t)=g(t) \cos \theta, \\
H_{\mathrm{eff}}^{(1)}=\epsilon\left[\frac{\hbar^{2}}{\mu} g \lambda^{-2} \cos \theta \nabla^{2}+\mathrm{i} \hbar \dot{g} \cos \theta\left(1+r \frac{\partial}{\partial r}\right)-\frac{\hbar^{2}}{2 \mu} g \lambda^{-2}\left(\cos \theta+2 \sin \theta \frac{\partial}{\partial \theta}\right)\left(\frac{1}{r^{2}}+\frac{1}{r} \frac{\partial}{\partial r}\right)\right] .
\end{gathered}
$$

Let us consider the case in which the gross evolution (the pantographic one) is associated to uniformly dilating box - this means $\lambda(t)=1+\kappa t$ - since in this case we know the solution of the relevant pantographic problem. Moreover, let us assume a smooth form for the function $g$, for example $g(t)=1-e^{-\gamma t}$. 


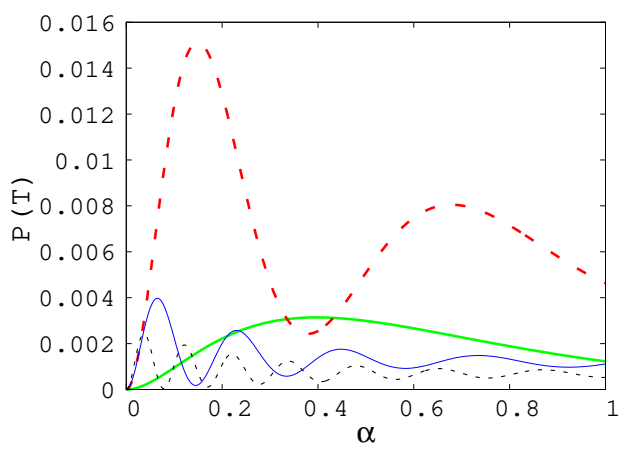

FIG. 1: (Color online). Populations of the states $\left|\psi_{1,1}\right\rangle$ (green bold solid line), $\left|\psi_{1,2}\right\rangle$ (red bold dashed line), $\left|\psi_{1,3}\right\rangle$ (blue solid line), $\left|\psi_{1,4}\right\rangle$ (black dashed line), when the initial state is $\left|\psi_{0,1}\right\rangle$. Here the time is expressed in $\kappa^{-1}$ units, $\hbar=1, \epsilon=0.05$ and $\gamma=5 \kappa$.

The energy eigenvalues and stationary states of a quantum particle confined in a circle of radius $r_{0}$ are given by [37]:

$$
\begin{aligned}
E_{m n} & =\frac{\hbar^{2}}{2 \mu r_{0}^{2}} a_{m n}^{2}, \\
\chi_{m n} & =(2 \pi)^{-1 / 2} A_{m n} J_{m}\left(k_{m n} r\right) \times e^{\mathrm{i} m \theta}, \quad m \in \mathbb{Z},
\end{aligned}
$$

where $J_{m}(x)$ is the Bessel function of $m$-th order, $a_{m n}$ is the $n$-th zero of $J_{m}$, and

$$
\begin{aligned}
& k_{m n}^{2}=\frac{2 \mu E_{m n}}{\hbar^{2}}=\frac{\left(a_{m n}\right)^{2}}{r_{0}^{2}} \Rightarrow k_{m n}=\frac{\left|a_{m n}\right|}{r_{0}}, \\
& A_{m n}=\left(\int_{0}^{r_{0}} r J_{m}\left(k_{m n} r\right)^{2} \mathrm{~d} r\right)^{-1 / 2} .
\end{aligned}
$$

It is very useful to note that in this case we have, for the matrix element $\left\langle\phi_{m n}(s)\left|H_{\text {eff }}^{(1)}(s)\right| \phi_{m^{\prime} n^{\prime}}(s)\right\rangle$, the selection rule $m=m^{\prime} \pm 1$. In the appendix $\AA$ we give the explicit expressions of the matrix elements involved in the perturbative treatment.

The selection rule is useful both as a simplification in the evaluation of the dynamics and as a warranty of the stability of the perturbation treatment, in this case. In fact, among the terms in Eq. (566) there are unbounded operators involving the variable $r$ (they are, $r^{-2}$ and $r^{-1} \partial_{r}$ ), but due to the behavior of the Bessel functions close to zero $\left(J_{m}(r) \sim r^{m}\right.$ when $\left.r \rightarrow 0\right)$, the only divergent matrix elements of the operators involving the radial variable are those between two $J_{0}$ 's: $\int_{0}^{1} r J_{0}\left(k_{0 n} r\right) J_{0}\left(k_{0 n^{\prime}} r\right) \mathrm{d} r$. Now, since such Bessel functions correspond to the same angular momentum $\left(m=m^{\prime}=0\right)$, the condition $m=m^{\prime} \pm 1$ is not fulfilled and the relevant matrix element of $H_{\text {eff }}^{(1)}$ is zero. Therefore $H_{\mathrm{eff}}^{(1)}$ is 'effectively bounded', in spite of the presence of unbounded operators related to the radial part.

In the following, we show some numerical calculations of the transition probabilities associated to specific initial conditions. We first consider the initial state $|\psi(t=0)\rangle=\left|\psi_{0,1}\right\rangle$ (the ground state), so that, at first order in the eccentricity, only transitions to states $\left|\psi_{ \pm 1, n}\right\rangle$ are allowed. In particular, in Fig 1 we show the transition probabilities to the states $\left|\psi_{1, n}\right\rangle$ with $n=1,2,3,4$. Due to the specific structure of the initial state and of the states of the basis, the transition probabilities to $\left|\psi_{1, n}\right\rangle$ and $\left|\psi_{-1, n}\right\rangle$ are equal. Moreover, in Fig 2 we show some of the transition probabilities evaluated assuming $\left|\psi_{2,1}\right\rangle$ as the initial condition.

\section{DISCUSSION}

In this paper we have extended the technique of Ref [8] to the cases of two-dimensional boxes. We have first of all reviewed the results related to the one-dimensional problem, and then we have studied in depth the case of a "free" particle confined in a two-dimensional box by mapping such problem into the one of a particle in a fixed domain but governed by a time-dependent Hamiltonian.

The two-dimensional problem reveals an important element of novelty with respect to the one-dimensional case, which is the role of the shape of the box (and its changing) through the whole process. In fact, the time-dependent Hamiltonian governing the dynamics of the particle in the picture associated to a fixed boundary is the sum of several 


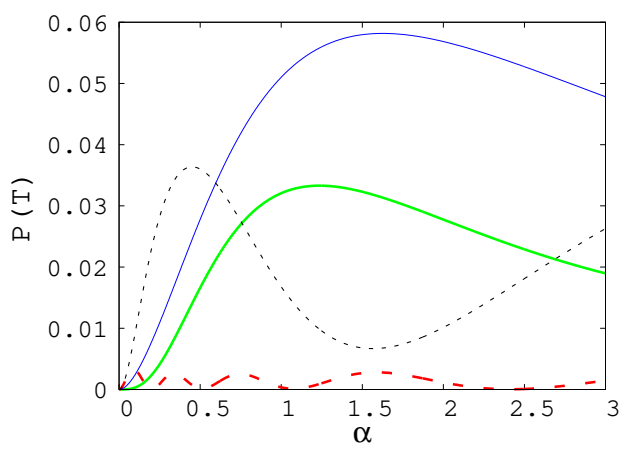

FIG. 2: (Color online). Populations of the states $\left|\psi_{3,1}\right\rangle$ (green bold solid line), $\left|\psi_{3,2}\right\rangle$ (red bold dashed line), $\left|\psi_{1,1}\right\rangle$ (blue solid line), $\left|\psi_{1,2}\right\rangle$ (black dashed line), when the initial state is $\left|\psi_{2,1}\right\rangle$. Here the time is expressed in $\kappa^{-1}$ units, $\hbar=1, \epsilon=0.05$, and $\gamma=5 \kappa$.

terms, many of which disappear when the boundary of the box changes its dimensions but not its shape (we have addressed this situation as the pantographic case). This has allowed to single out two classes of 2D problems depending on the presence of deformations of the contour of the box.

On the one hand we have shown that in the absence of deformation the results obtained in the one-dimensional case can be naturally extended. For example, the rate equation for the energy is just what one could guess starting from the rate equation of the energy in the one-dimensional moving box. Moreover, the time evolution of the particle in the case of uniformly moving boundaries is the very natural extension of the one-dimensional counterpart.

On the other hand when we consider deformation of the boundary, the situation becomes more complicated and the previously reported analogies to the one-dimensional case are no longer valid. In fact, the additional terms of the Hamiltonian coming from the deformation of the contour make the resolution of the relevant dynamical problem very difficult. We have then used an approach based on perturbation theory assuming the pantographic Hamiltonian as the unperturbed one, and the deformation terms, which play the role of perturbation, are assumed to be small enough. On this ground, we have shown that deformation of the boundary is responsible for transitions between pantographic states, which would not occur otherwise.

In order to conclude our analysis, we want to briefly comment on the fact that the methods and results reported for the $2 \mathrm{D}$ case can be easily extended to the case of a particle in a three-dimensional box. Indeed, in the $3 \mathrm{D}$ pantographic case, after performing the passage to the static boundary picture, the generator of time evolution turns out to be again the sum of the kinetic energy of a varying-mass particle and a dilation term very similar to the two-dimensional one. The complete dynamical solution that one can obtain when the velocity of the boundary is constant and the general energy rate equation (valid for any time dependence of the velocity of the walls) are the very natural extensions of the two-dimensional counterparts. Finally, if one considers also the presence of deformation, this can be treated perturbatively by following the same approach previously developed. Obviously, every calculation is more cumbersome, since it involves more terms than in the two-dimensional case.

\section{ACKNOWLEDGEMENTS}

We thank P. Facchi, G. Marmo and S. Pascazio for stimulating discussions on the subject of this paper. S.D.M. acknowledge support from the Italian National Group of Mathematical Physics (GNFM-INdAM).

[1] Klimchitskaya G L, Mohideen U, and Mostepanenko V M 2009 Rev. Mod. Phys. 811827

[2] Wilson C M, Duty T, Sandberg M, Persson F, Shumeiko V and Delsing P 2010 Phys. Rev. Lett. 105233907

[3] Fermi E 1949 Phys. Rev. 751169

[4] Doescher S W and Rice M H 1969 Am. J. Phys. 371246

[5] Pinder D N 1989 Am. J. Phys 5854

[6] Schlitt D W and Stutz C 1970 Am. J. Phys 3870

[7] Dodonov V V, Klimov A B and Nikonov D E 1993 J. Math. Phys. 343391

[8] Di Martino S, Anzà F, Facchi P, Kossakowski A, Marmo G, Messina A, Militello B, Pascazio S 2013 J. Phys. A 46 365301

[9] Lenz F, Liebchen B, Diakonos F K, Schmelcher P 2011 New J. Phys. 13103019 
[10] Mousavi S V 2013 Physics Letters A 3771513

[11] Mousavi S V 2012 EPL 9930002

[12] da Luz M G E and Cheng B K 1992 J. Phys. A: Math. Gen. 25 L1043

[13] Cheng B K and da Luz M G E 1993 Phys. Rev. A 474720

[14] da Luz M G E and Cheng B K 1994 Physica D: Nonlinear Phenomena 72244

[15] Schulman L S 1994 J. Phys. A: Math. Gen. 271703

[16] Zou J and Shao B 2000 Int. J. Mod. Phys. B 141059

[17] Gaveau B and Schulman L S 1986 J. Phys. A: Math. Gen. 191833

[18] Linington I E and Garraway B M 2008 Phys. Rev. A 77033831

[19] Xi Chen et al 2009 Phys. Rev. A 80063421

[20] Mousavi S V 2014 Phys. Scr. 89065003

[21] del Campo A and Boshier M G 2012 Sci. Rep. 2648

[22] del Campo A 2013 Phys. Rev. Lett. 111100502

[23] Messiah A 1995 Quantum Mechanics (Dover, Mineola)

[24] Traversa F L, Di Ventra M, Bonani F 2013 Phys. Rev. Lett. 110170602

[25] Moskalets M, Büttiker M 2002 Phys. Rev. B 66205320

[26] Shirley J H 1965 Phys. Rev. B 138979

[27] Aniello P 2005 J. Opt. B 7 S507

[28] Militello B, Aniello P, Messina A 2007 J. Phys. A: Math. Theor. 404847

[29] Zagury N, Aragao A, Casanova J, Solano E 2010 Phys. Rev. A 82042110

[30] Rigolin G, Ortiz G, Ponce V H 2008 Phys. Rev. A 78052508

[31] Hezhu Shao, Zhongcheng Wang 2009 Phys. Rev. E 79056705

[32] van Dijk W, Brown J, Spyksma K 2011 Phys. Rev. E 84056703

[33] Varga K 2012 Phys. Rev. B 85016705

[34] Konishi K and Paffuti G 2009 Quantum Mechanics: A New Introduction (Oxford: Oxford University Press)

[35] Sakurai J J 1993 Modern Quantum Mechanics (Addison Wesley)

[36] Kato T 1966 Perturbation Theory for Linear Operators (Springer-Verlag, Berlin). See Chapter VII, section 6.5, "Boundary perturbation."

[37] Robinett R W 1996 Am. J. Phys. 64440 


\section{Appendix A: Matrix Elements for the perturbative treatment}

In this appendix we give the explicit result of the evaluation of the matrix elements of $\hat{H}_{\mathrm{eff}}^{(1)}$ for a circular box which is deformed to an elliptical box and expanded.

Since

$$
\hat{H}_{\mathrm{eff}}^{(1)}=\hat{H}_{1}^{(1)}+\hat{H}_{2}^{(1)}+\hat{H}_{3}^{(1)},
$$

with obvious notation, we give separately the matrix elements of the three operators:

$$
\begin{aligned}
& \int_{0}^{t}\left\langle\phi_{m n}\left|H_{1}^{(1)}(s)\right| \phi_{m^{\prime} n^{\prime}}\right\rangle \mathrm{d} s=\epsilon\left(\delta_{m, m^{\prime}+1}+\delta_{m, m^{\prime}-1}\right) \times\left[2 F_{m n ; m^{\prime} n^{\prime}}^{(2)}(t) \times W_{m n ; m^{\prime} n^{\prime}}^{(2)}\right. \\
& \left.+F_{m n ; m^{\prime} n^{\prime}}^{(3)}(t) \times W_{m n ; m^{\prime} n^{\prime}}^{(3)}+2 F_{m n ; m^{\prime} n^{\prime}}^{(2)}(t) \times W_{m n ; m^{\prime} n^{\prime}}^{(4)}-\left(k_{m^{\prime} n^{\prime}}\right)^{2} F_{m n ; m^{\prime} n^{\prime}}^{(1)}(t) \times W_{m n ; m^{\prime} n^{\prime}}^{(2)}\right], \\
& \quad \int_{0}^{t}\left\langle\phi_{m n}\left|H_{2}^{(1)}(s)\right| \phi_{m^{\prime} n^{\prime}}\right\rangle \mathrm{d} s=\epsilon\left(\delta_{m, m^{\prime}+1}+\delta_{m, m^{\prime}-1}\right) \times \\
& \left.\quad F_{m n ; m^{\prime} n^{\prime}}^{(4)}(t) \times W_{m n ; m^{\prime} n^{\prime}}^{(1)}+F_{m n ; m^{\prime} n^{\prime}}^{(5)}(t) \times W_{m n ; m^{\prime} n^{\prime}}^{(3)}+F_{m n ; m^{\prime} n^{\prime}}^{(4)}(t) \times W_{m n ; m^{\prime} n^{\prime}}^{(4)}\right] \\
& \quad \int_{0}^{t}\left\langle\phi_{m n}\left|H_{3}^{(1)}(s)\right| \phi_{m^{\prime} n^{\prime}}\right\rangle \mathrm{d} s=\epsilon\left[\left(1 / 2+m^{\prime}\right) \delta_{m, m^{\prime}+1}+\left(1 / 2-m^{\prime}\right) \delta_{m, m^{\prime}-1}\right] \times \\
& {\left[F_{m n ; m^{\prime} n^{\prime}}^{(1)}(t) \times W_{m n, m^{\prime} n^{\prime}}^{(1)}+F_{m n, m^{\prime} n^{\prime}}^{(2)}(t) \times W_{m n, m^{\prime} n^{\prime}}^{(2)}\right]}
\end{aligned}
$$

where:

$$
\begin{aligned}
& F_{m n ; m^{\prime} n^{\prime}}^{(1)}(t) \equiv \frac{\hbar^{2}}{2 \mu} \int_{0}^{t} \frac{g(s)}{\lambda^{2}(s)} \operatorname{Exp}\left[i \xi_{m^{\prime} n^{\prime}}^{m n}(s)\right] \mathrm{d} s \\
& F_{m n ; m^{\prime} n^{\prime}}^{(2)}(t)=i \hbar \int_{0}^{t} g(s) \frac{\dot{\lambda}(s)}{\lambda(s)} \operatorname{Exp}\left[i \xi_{m^{\prime} n^{\prime}}^{m n}(s)\right] \mathrm{d} s \\
& F_{m n ; m^{\prime} n^{\prime}}^{(3)}(t)=-\mu \int_{0}^{t} g(s) \dot{\lambda}^{2}(s) \operatorname{Exp}\left[i \xi_{m^{\prime} n^{\prime}}^{m n}(s)\right] \mathrm{d} s \\
& F_{m n ; m^{\prime} n^{\prime}}^{(4)}(t) \equiv \frac{\mathrm{i} \hbar}{2} \int_{0}^{t} \dot{g}(s) \operatorname{Exp}\left[i \xi_{m^{\prime} n^{\prime}}^{m n}(s)\right] \mathrm{d} s \\
& F_{m n ; m^{\prime} n^{\prime}}^{(5)}(t)=-\frac{\mu}{2} \int_{0}^{t} \dot{g}(s) \lambda(s) \dot{\lambda}(s) \operatorname{Exp}\left[i \xi_{m^{\prime} n^{\prime}}^{m n}(s)\right] \mathrm{d} s
\end{aligned}
$$

with

$$
\xi_{m^{\prime} n^{\prime}}^{m n}(t)=\beta_{m^{\prime} n^{\prime}}(t)-\beta_{m n}(t)
$$

and

$$
\begin{aligned}
& W_{m n ; m^{\prime} n^{\prime}}^{(1)}=A_{m n} A_{m^{\prime} n^{\prime}} \int_{0}^{r_{0}} J_{m}\left(k_{m n} r\right)\left(\frac{1}{r}+\frac{\partial}{\partial r}\right) J_{m^{\prime}}\left(k_{m^{\prime} n^{\prime}} r\right) \mathrm{d} r . \\
& W_{m n ; m^{\prime} n^{\prime}}^{(2)}=A_{m n} A_{m^{\prime} n^{\prime}} \int_{0}^{r_{0}} r J_{m}\left(k_{m n} r\right) J_{m^{\prime}}\left(k_{m^{\prime} n^{\prime}} r\right) \mathrm{d} r \\
& W_{m n ; m^{\prime} n^{\prime}}^{(3)}=A_{m n} A_{m^{\prime} n^{\prime}} \int_{0}^{r_{0}} r^{3} J_{m}\left(k_{m n} r\right) J_{m^{\prime}}\left(k_{m^{\prime} n^{\prime}} r\right) \mathrm{d} r \\
& W_{m n ; m^{\prime} n^{\prime}}^{(4)}=A_{m n} A_{m^{\prime} n^{\prime}} \int_{0}^{r_{0}} r^{2} J_{m}\left(k_{m n} r\right) \frac{\partial}{\partial r} J_{m^{\prime}}\left(k_{m^{\prime} n^{\prime}} r\right) \mathrm{d} r .
\end{aligned}
$$

